\title{
The effect of number of stimulators on the optimal interstimulus onset interval in tactile apparent movement
}

\author{
JACOB H. KIRMAN \\ Queens College of the City University of New York, Flushing, New York 11367
}

\begin{abstract}
The effect of variations in the number of sequentially activated electromechanical stimulators on tactile apparent movement was examined in nine subjects. Judgments of successiveness or simultaneity were obtained as well. It was observed that four stimulators produced more frequent reports of apparent movement and resulted in optimal movement at shorter interstimulus onset intervals than did two stimulators, regardless of stimulus duration. Stimulus duration and interstimulus onset interval and their interaction were again found to be important determinants of tactile apparent movement.
\end{abstract}

In a recent article (Kirman. 1973). it was suggested that rapidly comprehensible tactile displays of speech or language should permit the perceptual integration of successively presented stimuli into relatively large linguistic units. It was also proposed that. if the proper spatial and temporal conditions were provided. the illusion of tactile apparent movement could serve as a mechanism for the integration of such stimuli into unitary percepts. However, requirements for the emergence of tactile apparent movement have not been determined for a sufficiently wide variety of stimulus conditions to provide practical guidelines for the designer of such tactile displays.

Most investigations of the phenomenon of tactile apparent movement have utilized two stimulators. whether vibratory or electrocutaneous. It has been established that the impressiveness of the illusion with two stimulators is influenced both by stimulus duration and by the interval between their onsets. It has further been shown that there is a considerable interaction between these two variables (Kirman. 1974a: Sherrick \& Rogers. 1966). However, since practical punctiform tactile displays will certainly utilize more than two successive stimuli, information is needed about the effects of increasing the number of sequential stimuli.

While it has been shown that increasing the number of successive stimuli enhances the impressiveness of the illusion of movement (Kirman, 1974b), the effect of such increases on the optimal temporal conditions for obtaining good movement has not been determined. The present experiment was designed to provide such information.

\section{METHOD}

\section{Subjects}

The subjects were 9 undergraduates at Queens College of CUNY. none of whom had had any prior experience with experiments using tactile stimulation or apparent movement. They were selected from an original group of 11 subjects. 2 of whom were eliminated from the experiment because of the unreliability of their judgments. All subjects were paid by the hour for their services.

\section{Apparatus}

The tactile stimulators that contacted the subjects' fingers were bronze rods. $.025 \mathrm{in} .(.64 \mathrm{~mm})$ in diam and rounded at the tip. that pushed up from 18 -in. $(3.2-\mathrm{mm})$ holes in a Plexiglas rest plate which served both to maintain the finger surface in a constant position relative to the stimulators and to minimize traveling waves on the skin surface between stimulus sites. These rods were direct extensions of the spring-loaded armatures of miniature soknoids (Electro-mechanisms Corp. SP-18) which were vertically mounted below the Plexiglas plate. Four of these solenoids were arranged in a straight line with a center-to-center distance of $.2 \mathrm{in} .(5.1 \mathrm{~mm})$ between adjacent bronze stimulators. They were activated by 1.5 -msec square-wave pulses of about $40 \mathrm{~V}$, which produced clearly felt. but not painful. "taps" on the skin. Since the inactive position of the bronze rods was below the surface of the Plexiglas plate. they made onls momentary contact with the skin at each pulse. Stimulus durations longer than a single 1.5 -msec pulse were produced by a train of such pulses occurring at a rate of $100 / \mathrm{sec}$. [For ease of presentation. all stimulus durations of two pulses or more will be referred to by the interval between the onset of the first pulse and the onset of the last pulse (omitting the constant additional duration of the last 1.5-msec pulse itself). For example, a stimulus described below as having a duration of $10 \mathrm{msec}$ consisted of two such pulses with an interval of $10 \mathrm{msec}$ between their onsets: a stimulus described as $20 \mathrm{msec}$ in duration consisted of three such pulses. etc. In addition. stimuli consisting of only one 1.5-msec pulse will be referred to below as having a duration of $1 \mathrm{msec}$ ] The activation of the appropriate solenoids at the proper intervals was controlled by a Datamec digital tape transport on which had been written as individual records each of the selected stimulus conditions.

\section{Procedure}

Of the $\mathbf{5 0}$ stimulus items to be judged. 25 used all four of the stimulators in the row [with an interstimulator distance of .2 in. $15.1 \mathrm{~mm})$ ]. While the remaining 25 items utilized only the two stimulators furthest apart in the row [with an interstimulator distance of .6 in. $(15.2 \mathrm{~mm})]$. For both the four-stimulator and the two-stimulator conditions. five stimulus durations $11,10.20 .50$. and $100 \mathrm{msec}$ ) were each combined with five interstimulus onset intervals $(30.50 .70 .90$. and $110 \mathrm{msec})$. Interstimulus onset interval (ISOI) refers to the temporal interval between the onsets of activation of stimulators within a stimulus item (this interval is sometimes designated in the literature as stimulus onset 
asynchony). In the case of the four-stimulator items. the ISOIs between each of the four successive component stimulators were the same within any given item. Similarly, the durations of activation of all stimulators were equal within any given item.

It should be noted that the term "stimulus duration" is used in this paper to refer to the length of time any individual solenoid stimulator was activated. Since complete stimulus items always comsisted of either tw'o or four stimulators sequentially energized at various 1SOIs. the overall length of time required for the presentation of any item was a function of the number of stimulators employed and the ISOI used, as well as the duration of the pulse train at each stimulator. For example, a four-stimulator itcm with an 1SOI of $50 \mathrm{msec}$ and a stimulus duration of $20 \mathrm{msec}$ would consist of the sequential energizing of the four solenoids with 50) msec between their onsets and with each solenoid remaining active for a duration of $20 \mathrm{msec}$. The entire item would require 170 msec for its presentation.

The set of 25 two-stimulator items was presented separately from the set of 25 four-stimulator items, and each set was provided with its own reference stimuli for the judgment of good movement, simultaneity, and successiveness. These procedures were designed to encourage subjects to adopt separate criteria for the judgment of the two sets of items.

During experimental sessions, the combinations of duration and ISOI were presented in random order within each set, and appropriate reference stimuli were presented before the 1 st item and after the 8 th and 16 th items. The reference stimuli for the iwo-stimulator set of items naturally utilized the two stimulators for that set. while the reference stimuli for the four-stimulator set used all four stimulators. The reference stimuli were all $100 \mathrm{msec}$ in duration, and had ISOls of $100 \mathrm{msec}$ for good movement, $0 \mathrm{msec}$ for simultaneity, and $310 \mathrm{msec}$ for successiveness.

The subject was seated. and his right index finger was positioned on the Plexiglas rest plate so that the two most distant stimulators 1.0 in. (15.2 mim) apart] would contact the fleshy pad at the distal end of the finger. At the beginning of the first experimental session. the subjects were exposed to several examples of the stimuli to be used in order to acquaint them with the experience of tactile apparent movement. They were instructed to try to avoid basing their judgments on duration, intensity, or "speed," but rather to concentrate on the impressiveness, smoothness, and continuity of the experience of movement. The subjects were asked to judge the stimuli as follows: "A" if they experienced movement that was impressive and continuous; " $B$ " if movement was experienced as unimpressive or discontinuous; " $C$ " if the movement was hardly perceivable or ambiguous: and "zero" if they definitely experienced no movement. In the latter case, the subjects were asked to specify whether the stimuli appeared to be simultaneous or successive.

Each experimental session consisted of one set of judgments of all stimuli. with the order of presentation of the groups of two- and four-stimulator conditions alternated from session to session. There were six such sessions in all.

\section{RESULTS AND DISCUSSION}

Since the subjects judged each stimulus according to several criteria of movement ("A," "B," or "C"), it was possible to analyze the data on movement judgments in several ways. A preliminary analysis revealed that essentially the same results were obtained regardless of whether only " $A$ " judgments or both " $A$ " and " $B$ " judgments pooled together were taken as the criterion level for a definition of movement. Since the higher criterion ("A" judgments only) reveal these results at least as clearly as the alternatives, only the data from that analysis will be presented here. For the remainder of this paper. therefore. "movement judgments" refer only to those that were judged by the subjects as impressive and continuous movement.

For each subject, the percentage of trials on which such movement was reported was determined for each stimulus condition. The averages of these percentage scores across subjects are plotted in Figure 1, which gives one graph for the 25 two-stimulator conditions and one for the 25 four-stimulator conditions. In both graphs. the percentages are plotted as a function of ISOI for each stimulus duration. Before examining the effects of number of stimulators on the temporal conditions for tactile apparent movement, the present results will first be compared with previous findings.

The two-stimulator graph presents what is essentially a replication of a previous investigation of the effects of ISOI and duration on tactile movement with two stimulators. That study (Kirman, 1974a) reported that as ISOI was increased, movement judgments increased to a maximum value and then decreased. It was further observed that, as duration was increased, the ISOI value for optimal movement shifted toward higher values and the average movement score increased for all ISOIs. Both graphs
Figure 1. Percentage of trials, averaged across subjects, on which movement was reported for each stimulus condition. Data are plotted as a function of ISOI for each of five durations in two graphs, one for all twostimulator conditions and one for all fourstimulator conditions.
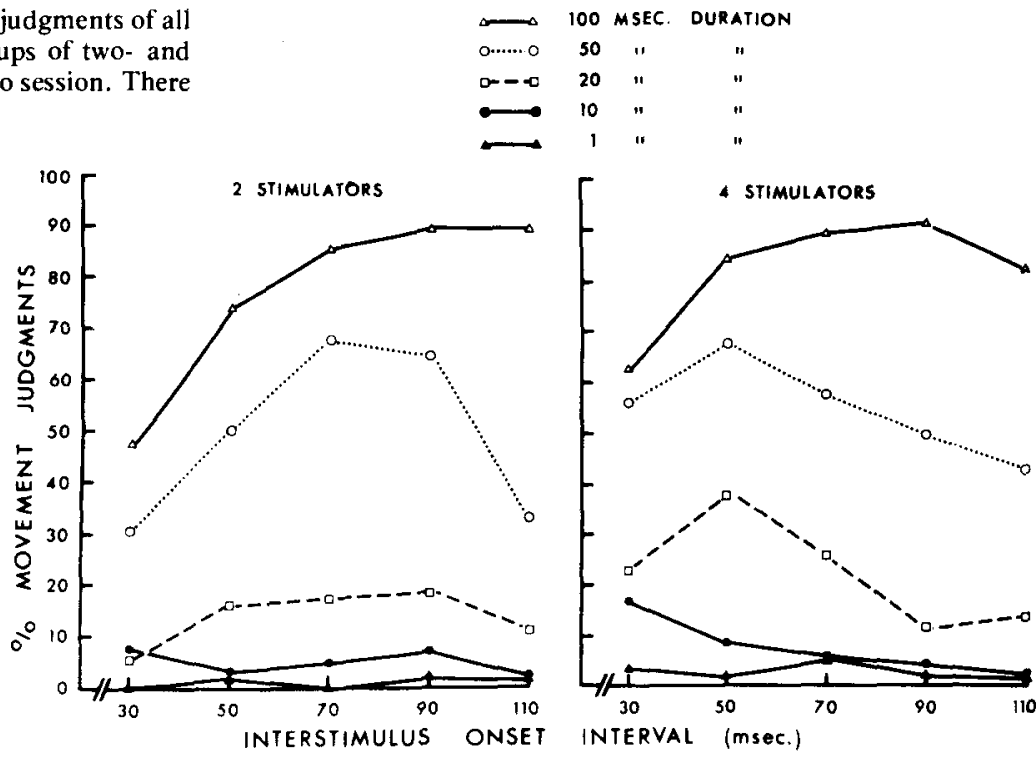


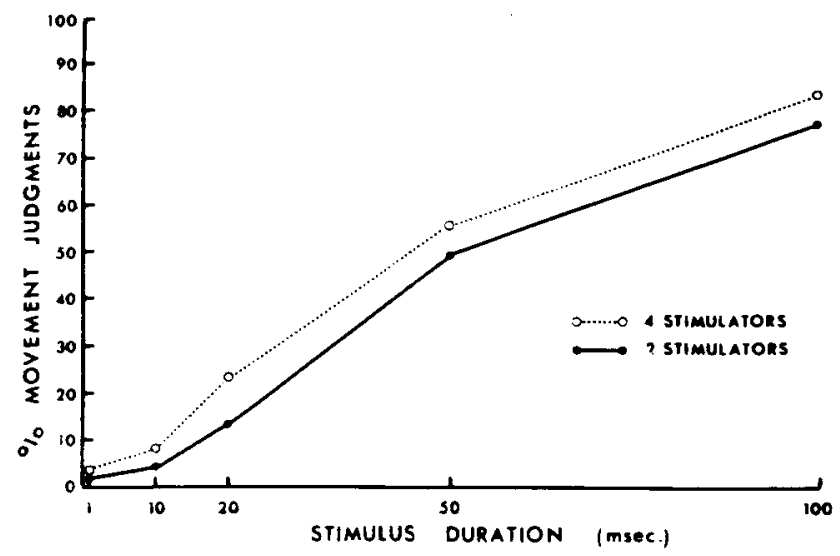

Figure 2. Percentage of triak, averaged actoss subjects and ISOIs, on which movement was reported for each combination of duration and number of stimulators.

of Figure 1 show that similar effects were obtained in the present data. With the exception of the two lowest duration curves. which appear to be confined by floor effects. an optimum ISOI value is indicated for each curve. An analssis of variance performed on both the two- and four-stimulator conditions combined showed a significant main effect due to ISOI $[F(4,32)=2.87$. $\mathrm{p}<.05$ ]. Figure 1 also suggests that optimum ISOIs shift toward higher values as duration is increased for both the two- and the four-stimulator conditions. This shift was statistically supported in the above analysis of variance by a significant interaction between duration and ISOI $[F(16.128)=4.57, p<.005]$. As a further check, an additional analysis of variance was performed on individual optimal ISOIs, determined separately for each subject. for each duration of both the two- and four-stimulator conditions. Here, also. the effect of duration on the value of ISOI optima was signiticant $[F(4.32)=11.98, p<.005]$. It is clear as well from Figure 1 that. with longer durations, higher percentages of movement were given for almost every value of ISOI for both the two- and the four-stimulator conditions. This effect of duration on apparent movement is plotted more directly in Figure 2. in which movement scores were averaged over all ISOIs for each duration. Analysis of variance showed this to be a highly significant effect $[F(4,32)$ $=109.18 . \mathrm{p}<.005]$. The present results then contirm in all essentials the findings of the earlier study.

Turning to the effects of number of stimulators, it should be noted that the present procedure encouraged subjects to adopt separate criteria for the evaluation of apparent movement for the two- and the four-stimulator conditions. This was done because of the results of a previous experiment, which had encouraged subjects to use the same criterion when judging a wide variety of stimuli that differed in number of stimulators as well as duration and ISOI (Kirman. 1974b). Although that procedure served well to evaluate the effect of number of stimulators on the impressiveness of tactile apparent movement over a wide range of ISOIs and durations, it did not permit the location of optimal values of ISOI for each condition of number of stimulators and duration. It appeared that the criterion adopted for judgment of movement was too gross to reveal differential ISOI effects. and it was not possible to determine the effect of variations in the number of stimulators on such optima.

It was expected that the procedure used in the present experiment would permit subjects to make finer distinctions within each of the two sets of stimuli that differed in number of stimulators. and therefore that optimal ISOIs would be observable within each set. This adoption of independent criteria for the two sets of stimuli would. of course. decrease the sensitivity of observations of differences between the two sets in the percentage of movement judgments overall.

It can be seen from Figure 2 that the four-stimulator condition nonetheless resulted in consistently higher percentages of movement reports at all durations than did the two-stimulator condition. and this effect of number of stimulators was statistically significant $[F(1.8)=7.22, p<.05]$, in agreement with the earlier study referred to (Kirman. 1974b)

As had been hoped, differential effects due to ISOIwere observed within each of the two sets of stimuli. Figure 1 shows that at least for the three longest duration curves in both sets, relative ISOI optima are indicated. The two 50-msec curves for both two and four stimulators show clear maxima, and it is evident that the optimal ISOI for the two-stimulator condition at this duration is at $70 \mathrm{msec}$ or above. while the optimal ISOI for the equivalent four-stimulator condition is around $50 \mathrm{msec}$. The 100 - $\mathrm{msec}$ curves show the same trend, with the four-stimulator curve peaking at around $90 \mathrm{msec}$. while the two-stimulator curve is still at an apparent maximum at $110 \mathrm{msec}$. Sinilarly. the 20-msec curve for four stimulators shows a maximum at $50 \mathrm{msec}$, and the $20-\mathrm{msec}$ two-stimulator curve has a weakly indicated maximum in the vicinity of $90 \mathrm{msec}$. When the curves for all five durations were averaged for the two- and four-stimulator conditions. respectively, they yielded the curves shown in Figure 3 . It is clear from these two curves that tactile apparent movement is more impressive at lower values of ISOI for four stimulators than for two stimulators. with the optimal value of ISOIfor four stimulators being $50 \mathrm{msec}$ and the optimal value for two stimulators being $90 \mathrm{msec}$. Analysis of variance indicated that the interaction between ISOI and the number of stimulators was signiticant $[\mathrm{F}(4.32)=4.00 . \mathrm{p}<.01]$.

In order to further check on the effect of number of stimulators on ISOI optima. the individual optimal ISOIs that were determined separately for each 


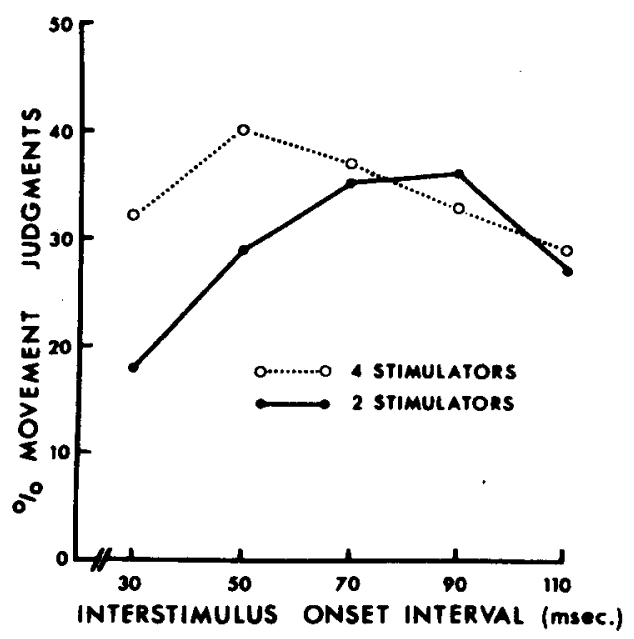

Figure 3. Percentage of trials, averaged across subjects and durations, on which movement was reported for each combination of ISOI and number of stimulators.

subject were examined. Here also, the four-stimulator conditions, consistently yielded the best movement at lower values of ISOI than did the two-stimulator conditions. The additional analysis of variance done on these individually determined optima showed a significant effect of number of stimulators on the optimal value of ISOI $[F(1,8)=4.69, p<.05]$.

It should be noted that the variable referred to here as "number of stimulators" included not only variations from two to four stimulators, but also variations in interstimulator spacing from .6 in. $(15.2 \mathrm{~mm})$ to $.2 \mathrm{in} .(5.1 \mathrm{~mm})$, although it is true that the overall end-point to end-point distance was .6 in. $(15.2 \mathrm{~mm})$ for both sets of stimuli. The variable is therefore confounded (and inevitably so, since if interstimulator spacing had been kept constant, then the end-point to end-point distance would have covaried with number of stimulators). It is therefore not possible on the basis of the present study alone to decide whether it is the variation in the number of stimulators or the difference in interstimulator spacing that is responsible for the observed effects. However, in view of previous observations with the same equipment that revealed hardly any effects on tactile apparent movement of variations in interstimulator distance from .2 to .6 in. (5.1 to $15.2 \mathrm{~mm}$ ) (Kirman, 1974b), and further, in view of the report of Sherrick and Rogers (1966) that large variations in distance did not influence the value of the optimal ISOI, it is likely that the effects reported here are primarily due to the variation in number of stimulators rather than distance.

Although there apparently have been no previous
Figure 4. Percentage of trials, averaged across subjects, on which judgments of successiveness and simultaneity were made for each stimulus condition. Data are plotted as a function of ISOI for each of two numbers of stimulators in five graphs, one for each stimulus duration.
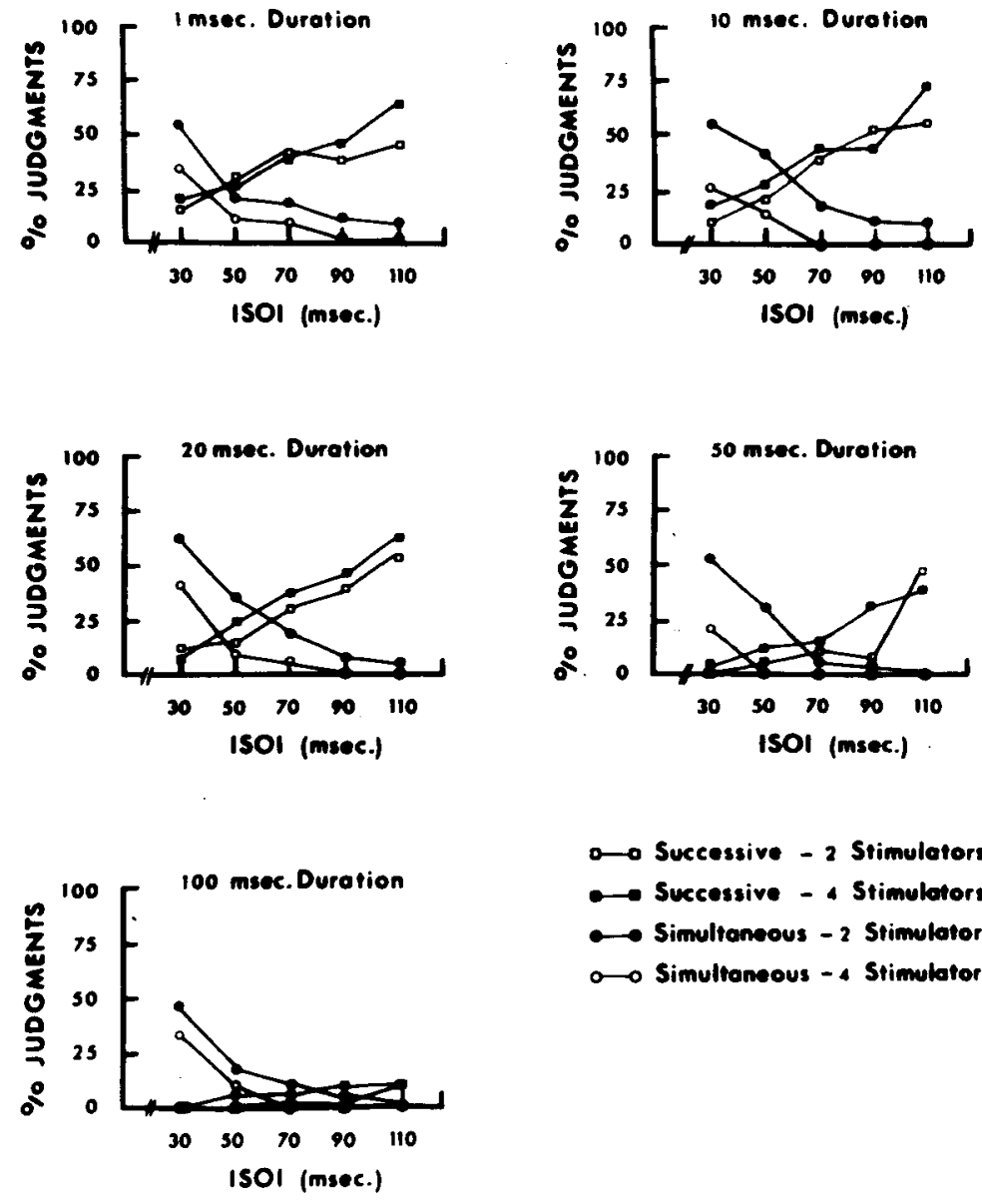
reports that increasing the number of stimulators lowers the value of the optimal ISOI. it has previously been observed that such an increase in number of stimulators broadens the range of ISOIs over which good movement can be obtained (Kirman. 1974b; Sherrick, 1968). Such a broadening. however, is not evident in the present data (see Figure 1). It is likely that such reported effects have been due to the fact that increasing the number of stimulators markedly increases the impressiveness of tactile apparent movement at all values of ISOI. and if subjects adopt a sufficiently modest subjective criterion for good movement. a wider range of ISOIs will meet that criterion as the number of stimulators is increased.

With respect to the second temporal variable investigated. stimulus duration, increasing the number of stimulators has no influence on the effects of duration on apparent movement. It can be seen from Figure 2 that the curve relating movement reports to duration for two stimulators is virtually parallel to the same curve for four stimulators. Analysis of variance found this interaction (number of stimulators and duration) to be not significant $[F(4.32)=.85]$. Although the three-way interaction between number of stimulators, duration, and ISOI was found to be barely significant $[F(16.128)=1.73$, $p<.05]$ in the major analysis of variance, it is perhaps unnecessary to speculate on the meaning of this complex interaction. since it was not found to be significant in the analysis of variance performed on the individual ISOI optima separately determined for each subject. Although, as has been discussed, both number of stimulators and duration each had significant main effects on these optimal ISOIs, the interaction between the two variables was not significant $[\mathrm{F}(4.32)=.54]$.

The findings with respect to judgments of successiveness and simultaneity are in complete agreement with earlier reports'(Kirman, 1974a, b). As Figure 4 show's, increasing ISOI naturally decreases simultaneity judgments $[F(4.32)=53.99$. $p<.005]$ and increases judgments of successiveness $[F(4.32)=24.35, p<.005]$. Increasing stimulus duration markedly decreases successiveness judgments at all values of ISOI $[F(4.32)=9.75, p<.005]$ but has no effect on simultaneity judgments $[F(4,32)=.831$. Increasing the number of stimulators from two to four produces a considerable decrease in judgments of simultaneity for all durations and ISOIs $[F(1.8)=18.08$, $p<.005]$. but has no significant effect on successiveness judgments $[F(1,8)=2.23, \mathrm{p}>.10]$.

The main finding of this experiment is that optimal values of ISOI are lowered as the number of sequentially activated stimulators is increased from two to four. This result suggests that the designer of tactile displays who wishes to create an illusion of tactile apparent movement from a succession of many stimuli not only can, but should, present stimulil at a considerably faster rate than would be indicated by previous apparent movement studies utilizing only two stimulators. Since a persistent concern among the designers of tactile displays is the relative slowness that has been associated with all existing tacile codes, this is an encouraging result.

\section{REFERENCES}

Kirmax. J. H. Tactile communication of speech: A revieu and an analysis. Psychological Bulletin. 1973. 80, 54-74.

Kirmax. J. H. Tactile apparent movement: The effects of interstimulus onset interal and stimulus duration. Perception \& Psichophisics. 1974. 15. 1-6. (a)

Kirman. J. H. Tactile apparent movement: The effects of number of stimulators. Journal of Experimental Psychology. 1974. 103. 1175-1180. (b)

SHERRICK. C. E. Studies of apparent tactual movement. In D. R. Kenshalo (Ed.). The skin senses. Springfield. Ill: Thomas. 1908.

Sherrick. C. E.. \& Rogers, R. Apparent haptic movement. Perception \& Psychophysics, 1966, 1, 175-180.

(Received for publication February 19. 1974; revision accepted October 21.1974.$)$ 